\title{
Deformable particles with anisotropic interactions: unusual field-induced structural transitions in ultrasoft ionic microgel colloids $\dagger$
}

\author{
Priti S. Mohanty, ${ }^{a b}$ Anand Yethiraj ${ }^{* c}$ and Peter Schurtenberger ${ }^{a}$
}

Ionic microgels are intriguing soft and deformable colloids with an effective pair potential that crosses over from Yukawa-like at large distances to a much softer repulsive interaction at short distances. Here we report the effect of adding an anisotropic dipolar contribution to colloids with such "ultra-soft" interactions. We use an alternating electric field to induce a tunable dipolar contribution, and study the resulting particle self-assembly and phase transitions in situ with confocal laser scanning microscopy. We find significant field-induced structural transitions at low as well as at very high effective volume fractions. At $\phi_{\text {eff }}=0.1$ we observe a transition from an isotropic to a string fluid. At $\phi_{\text {eff }}=\mathbf{0 . 8 5}$, there is a reversible transition from an amorphous to a dipolar crystalline state, followed by the onset of a gas-(string) solid coexistence. At $\phi_{\text {eff }}=1.6$ and 2.0, i.e. far above close packing, evidence for a field-induced arrested phase separation is found.

Soft colloids, where the interparticle distance $a_{\mathrm{s}}$ can be smaller than the particle diameter $\sigma$, and where the interaction potential shows a finite repulsion at or beyond contact, have recently attracted considerable interest from the experimental and theoretical soft matter community. ${ }^{1-6}$ In particular, cross-linked microgels such as poly $(N$-isopropylacrylamide) (PNIPAm) have been used frequently $^{7-13}$ as excellent model systems for soft colloids. As a result of the soft potential, microgels can be packed to give an effective volume fraction $\phi_{\text {eff }}$ far above closed packing $\phi_{\mathrm{cp}}$, with enormous consequences for the resulting structural and dynamic properties. ${ }^{10,12}$ Ionic microgels are particularly intriguing model systems in this context, as recent theoretical ${ }^{3,4}$ and experimental ${ }^{14,15}$ studies have demonstrated that the effective interaction potential crosses over from a Yukawa type interaction at $a_{\mathrm{s}} \gg \sigma$ to a much softer one at $a_{\mathrm{s}} \leq \sigma$. This soft-repulsive interaction is expected to dominate at high $\phi_{\text {eff, }}$ and to give rise to an extremely rich phase behavior with various new crystalline phases appearing. ${ }^{3,4}$

There is increasing interest in colloidal systems with non-centrosymmetric interaction potentials. Approaches include short-range

${ }^{a}$ Division of Physical Chemistry, Lund University, Lund, Sweden

${ }^{b}$ Department of Chemistry, University of Fribourg, Fribourg, Switzerland 'Department of Physics and Physical Oceanography, Memorial University, St.John's, NL, Canada.E-mail: ayethiraj@mun.ca anisotropic interactions, such as those in Janus or "patchy" colloids, ${ }^{16-18}$ and long-ranged anisotropic contributions to hardsphere-like systems through the application of an external electric field. ${ }^{19-23}$ Previous studies on different colloids in aqueous or partially polar media have clearly shown that the dipolar interaction is the dominant field-induced interaction at high frequencies. ${ }^{19,21-24}$ An external field applied to hard-sphere suspensions induces structural transitions from an isotropic to a string fluid at low densities, and to body-centred tetragonal crystallites at higher packing fractions. ${ }^{2,19-23,25-27}$ For colloids with soft, electrostatic repulsive interactions an interplay between long-range electrostatic repulsion and the external field gives many non-close-packed crystalline structures. ${ }^{23,24}$ We thus expect that adding anisotropy to the interaction potential between soft, deformable particles should lead to an even more complex phase behavior.

Microgel colloids exhibit glassy behavior at zero fields and high packing. The competition between crystallization and vitrification has been studied in hard-sphere systems, ${ }^{28-30}$ and while local re-arrangements during the aging process have been studied experimentally, ${ }^{31}$ the detailed microscopic mechanism of the glass-to-crystal transition remains unclear. A model system that could exhibit fielddriven transitions out of an amorphous state could thus be used to understand these microscopic mechanisms.

A study of such a system could also have other implications. Dipolar colloids have been explored extensively as possible electrorheological materials ${ }^{20,32}$ that display a field-induced yield stress, but these yield stresses are too small in hard-sphere suspensions to be practical in applications. Larger yield stresses have recently been obtained in colloids with significant dielectric constant heterogeneities, with the surface playing an important role. ${ }^{33,34}$ PNIPAm-based microgel particles have a very large surface area. As a result of their thermoresponsive nature, their dielectric constant and concomitantly their electric field response are expected to be temperature sensitive ${ }^{35}$ (this is demonstrated in this work in the ESI, Fig. S3†). Moreover, ionic microgels usually contain a large amount of confined counterions, which should result in strong field effects due to conductivity contributions. Moreover, ionic microgels usually contain a large amount of confined counterions with a possible non-uniform distribution ${ }^{36}$ due to a difference in density between the dense core and the fuzzy shell. This could lead to an interesting field effect due to an additional conductive contribution.

Here we report on the first study of ionic microgel dispersions in their swollen state (at $T=20^{\circ} \mathrm{C}$ ) in the presence of an alternating 
(AC) electric field. These studies were carried out in two different regimes: (a) $\phi_{\text {eff }}=0.1 \ll \phi_{\text {cp }}$, where the long-range screened Coulomb potential dominates, and (b) $\phi_{\text {eff }}=0.85,1.6$, and $2.0 \gg$ $\phi_{\mathrm{cp}}$, where the soft-repulsive interaction dominates. An overview of our observations is shown in Fig. 1. At low packing $\left(\phi_{\text {eff }}=0.1\right)$, a transition from isotropic to string fluid was observed, qualitatively consistent with field effects in colloids with hard-sphere or electrostatic repulsions. ${ }^{22,23}$ Above the close packing limit, at $\phi_{\text {eff }}=0.85$, we observed a transition from a disordered (glass-like) phase to a dipolar ordered phase. At ultra-high packing fractions $\left(\phi_{\text {eff }}=1.6\right.$ and 2.0), we found field induced aggregation or phase separation at a very low field strength, consistent with an electric-field-driven transition from a repulsive to an attractive glass.

Zero-field behavior - Ionic PNIPAm microgels were synthesized by free-radical precipitation polymerization. ${ }^{7,9}$ Initial characterization was carried out by static light scattering in the swollen state at $20{ }^{\circ} \mathrm{C}$. The results shown in Fig. 2 are consistent with a core-shell structure following the "fuzzy sphere" model, ${ }^{8}$ with a core radius $R_{\text {core }}=586 \mathrm{~nm}$, a shell thickness $2 \sigma_{\text {surf }}=78 \mathrm{~nm}$, a hydrodynamic radius $R_{\mathrm{h}}=724 \mathrm{~nm}$, and a polydispersity of $10 \%$. Details of the particle synthesis and characterization are provided in the ESI, Fig. S1 and text. $\dagger$

Concentrated suspensions were imaged using confocal laser scanning microscopy (CLSM) in the absence of an external electric field in order to assess their intrinsic interaction potential. Measurements were made at a temperature of $20^{\circ} \mathrm{C}$ with either an upright CLSM (Nikon Eclipse 80-i with $\mathrm{C} 1$ point scanner, excitation wavelength $\lambda=$ $561 \mathrm{~nm}$ ) or an inverted CLSM (Leica DMI6000 and SP5 tandem scanner in the resonant mode at 50 frames per second, excitation wavelength $\lambda=543 \mathrm{~nm}$ ), and $60 \times$ and $63 \times$ immersion objectives with a numerical aperture of 1.4 .

All measurements were made more than 10 particle diameters away from the cover slide to minimize wall effects. The resulting images were analyzed using standard methods, ${ }^{37,38}$ and pair correlation functions $g(r)$ were determined. A typical example at $\phi_{\text {eff }}=0.2$ is shown in Fig. 2B together with a theoretical calculation for particles interacting

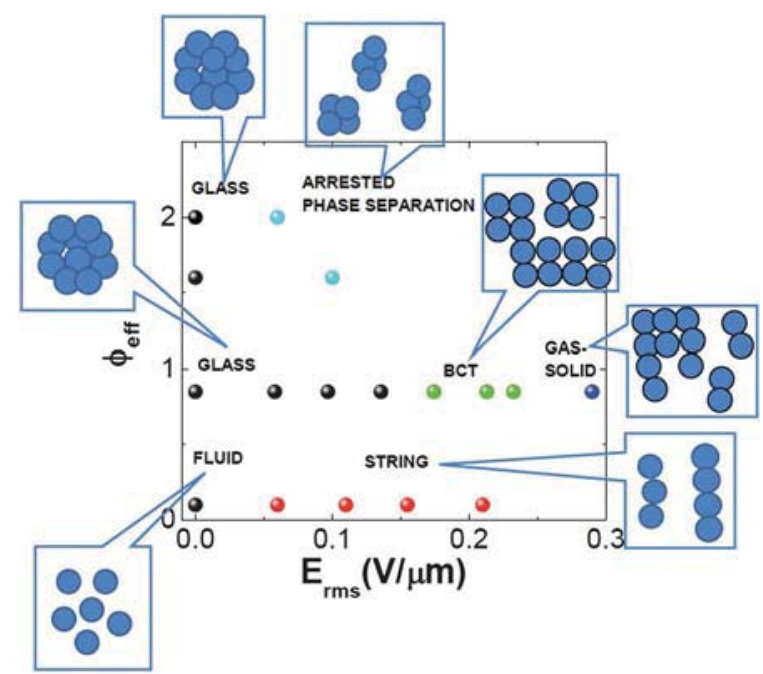

Fig. 1 Phase behavior of ionic microgels in the presence of an alternating electric field exhibits not only the fluid-string-BCT phase sequence seen for hard spheres at low packing, but also novel glass to crystal and to arrested dynamical states at higher packing.
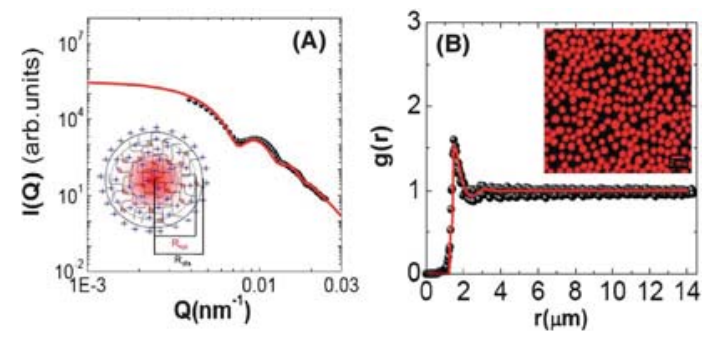

Fig. 2 (A) Static light scattering intensity versus the scattering vector, $I(Q)$ vs. $Q$, for a dilute microgel suspension together with a fit using the fuzzy sphere model. The inset shows a schematic representation of the ionic microgel with the different radii obtained. (B) Pair correlation function $g(r)$ for a microgel suspension at $\phi_{\text {eff }}=0.2$, obtained in the fluid state using CLSM (typical image shown in the inset) together with a calculation based on a Yukawa potential.

with an effective pair potential of a Yukawa form $U_{\text {eff }}(r)=\frac{Z_{\mathrm{eff}}^{2} e^{2}}{4 \pi \varepsilon \varepsilon_{0}}\left[\frac{\exp (\kappa R)}{1+\kappa R}\right]^{2} \frac{\exp (-\kappa r)}{r}$. Here we used $R=650 \mathrm{~nm}$, an effective charge $Z_{\text {eff }}=400 e$ per microgel and a Debye screening length $\kappa^{-1}=175 \mathrm{~nm}$, and the calculation was performed using liquid state theory based on the $\mathrm{HNC}$ closure relation (note that the overall bare charge on the particles is much larger, resulting from a large number of confined internal counterions, as was verified by previous titration experiments on ionic (PNIPAM-co-PAA) microgels ${ }^{14}$ ). The experimental $g(r)$ is quantitatively reproduced except for short distances $r<$ $2 R$, where we see small but systematic deviations that are most likely due to contributions from the intrinsic softness of the microgels and the finite resolution of the CLSM. Fig. 2B demonstrates that the interactions between the particles at distances $r>2 R$ are dominated by weakly screened electrostatic interactions, resulting in a phase behavior that mimics that of charged colloids up to fairly high volume fractions, when the softness of the particles becomes important. ${ }^{3,4}$

Field effects at low $\phi_{\mathrm{eff}}$ - Experiments were carried out at $\phi_{\mathrm{eff}}=0.1$ at a frequency of $100 \mathrm{kHz}$. Two-dimensional (2d) images at different electric field strengths $E$ (in $\mathrm{V}_{\mathrm{rms}} \mu \mathrm{m}^{-1}$, with the field applied in the image plane, along the $X$-axis) are shown in Fig. 3, top row. The samples were contained between two cover slides separated by a 70 $\mu \mathrm{m}$ spacer. One cover slide was coated with indium tin oxide (ITO) with a $700 \mu \mathrm{m}$ sample gap etched out, which ensures a homogeneous field in the sample area where CLSM experiments were performed. At field strengths as low as $0.06 \mathrm{~V}_{\text {rms }} \mu \mathrm{m}^{-1}$, the particles associate into chains that orient along the field direction, with strings coexisting with single particles, and breaking and reforming due to competition between thermal fluctuations, repulsion and attractive dipole-dipole interactions. Upon increasing $E$, chains grow in length and stiffen, and intra-chain particle-particle distances $a_{\mathrm{s}}$ decrease. The entire process is field-reversible for all field strengths investigated.

It is interesting to compare the field effects to those previously observed for a hard-sphere-like PMMA colloidal system, ${ }^{23}$ where the interaction potential was a combination of a hard sphere contribution, a soft Yukawa repulsion and an induced dipole attraction of variable strength. For the PMMA system, $a_{\mathrm{s}}$ also decreased upon increasing field strength, but $a_{\mathrm{s}}$ was limited to a value greater than or equal to the particle diameter $\sigma$. This is quite different for the microgels, where the intrinsic softness of the particles allows for values of $a_{\mathrm{s}} / \sigma<1$, and we find $a_{\mathrm{s}} / \sigma \approx 0.75$ at $0.21 \mathrm{~V}_{\mathrm{rms}} \mu \mathrm{m}^{-1}$. In principle, knowing the strength of the dipolar attraction in our 


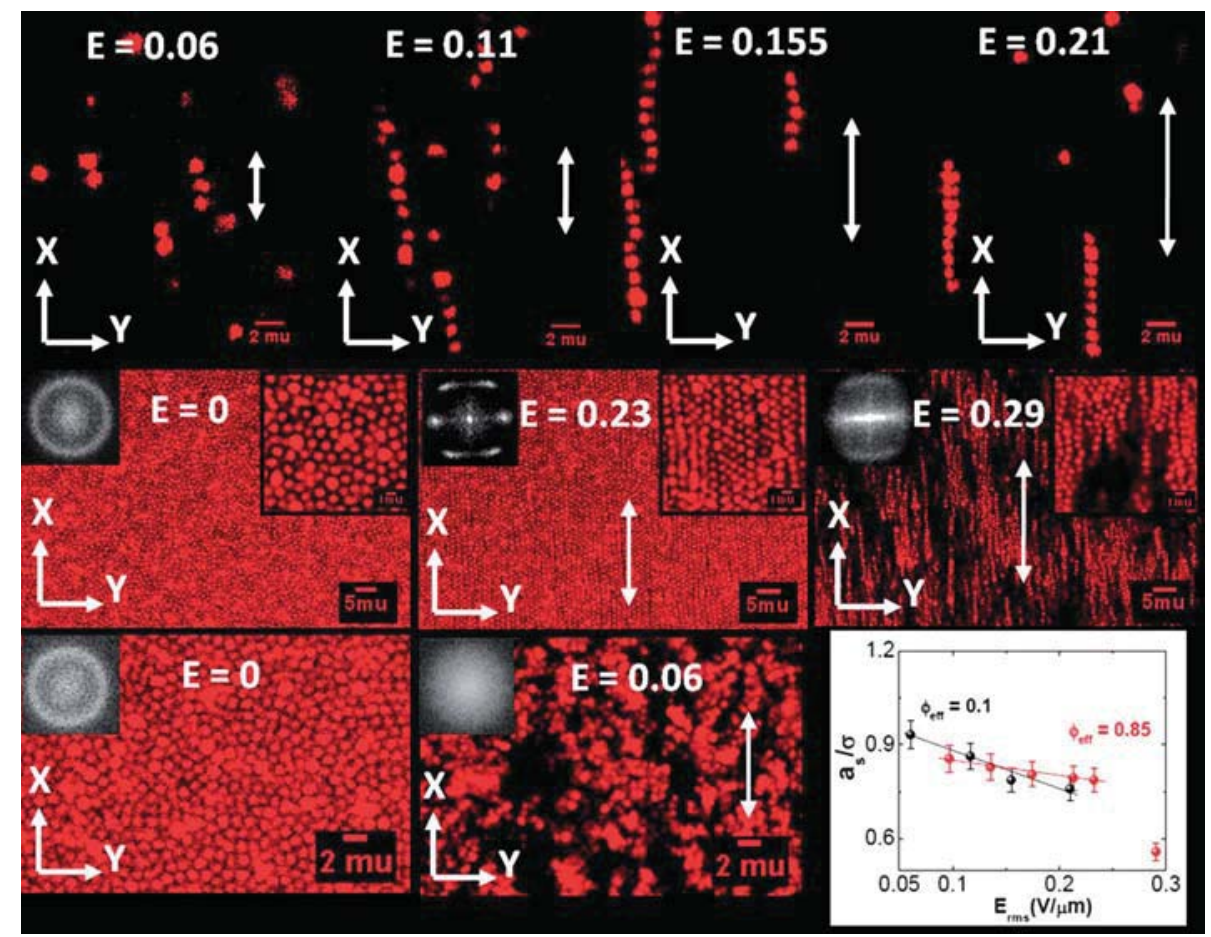

Fig. 3 Top: microgels at $\phi_{\text {eff }}=0.1$ in an applied electric field. $2 \mathrm{~d}$ confocal images taken in the bulk (10 particle diameters from the cover glass surface) at $E=0.06,0.11,0.155,0.21 \mathrm{~V}_{\mathrm{rms}} \mu \mathrm{m}^{-1}$. Middle: microgels at $\phi_{\mathrm{eff}}=0.85$, images at $2 \times$ and right insets at $8 \times$ zoom, in an applied electric field $E=0.0,0.23$, $0.29 \mathrm{~V}_{\mathrm{rms}} \mu \mathrm{m}^{-1}$ respectively. Bottom: microgels at $\phi_{\mathrm{eff}}=2.0$, images at $3 \times$ zoom, at $E=0.0,0.06 \mathrm{~V}_{\mathrm{rms}} \mu \mathrm{m}^{-1}$, and a plot of $a_{\mathrm{s}} / \sigma$ as a function of $E$ for $\phi_{\mathrm{eff}}=0.1,0.85 . a_{\mathrm{s}}$ is the average distance between particles in a chain and $\sigma$ is the zero-field diameter in the swollen state. In all images, the two-headed arrow indicates the field direction and the left insets show the Fourier transforms of the corresponding images.

experiments we could directly measure the soft repulsive potential between ionic microgels at distances below contact. Unfortunately, the fact that the particles contain a large number of trapped but mobile counterions and a complex density profile makes this a difficult task, and we can currently only provide the phenomenological relationship $a_{\mathrm{s}} v s$. $E_{\mathrm{rms}}$ shown in the last panel of Fig. 3. At higher field strengths $\left(E=0.25 \mathrm{~V}_{\mathrm{rms}} \mu \mathrm{m}^{-1}\right)$, the strings start to associate laterally to form aggregates (data not shown), similar to but less well ordered than those seen in the case of hard spheres. ${ }^{22}$

Field effects at high $\phi_{\text {eff }}-$ We next looked at the field response at very high volume fractions $\left(\phi_{\text {eff }}>\phi_{\mathrm{cp}}\right)$. Under these conditions $\left(a_{\mathrm{s}} / \sigma<\right.$ 1), short-range soft-repulsive interactions dominate, and a glass-like structure has been reported in the absence of an external field ${ }^{14}$ (see also Fig. S2 in the ESI $\dagger$ ). This is a concentration regime that is not accessible for dipolar hard spheres, and thus no previous data exist. For $\phi_{\text {eff }}=0.85$, we varied $E$ from 0 to $0.3 \mathrm{~V}_{\mathrm{rms}} \mu \mathrm{m}^{-1}$. With increasing field strength, we observed a re-entrant order-disorder transition (Fig. 3, middle row). An initial field response occurred around $E=$ $0.17 \mathrm{~V}_{\mathrm{rms}} \mu \mathrm{m}^{-1}$, where the particles started to form strings along the field direction. Ordering thus occurs at higher field strengths compared to the observations made at lower $\phi_{\text {eff }}$, consistent with the fact that now a larger dipolar force is required to overcome the arrested dynamics in the glassy state. Clearly ordered structures formed by well-aligned parallel strings could be observed between 0.19 and $0.29 \mathrm{~V}_{\mathrm{rms}} \mu \mathrm{m}^{-1}$. At $E_{\mathrm{rms}}=0.29 \mathrm{~V}_{\mathrm{rms}} \mu \mathrm{m}^{-1}$, the strings show a greater lateral overlap with a dramatically decreased value of $a_{\mathrm{s}} / \sigma$ and form larger aggregates, thus creating additional free space that contains some individual strings. This is akin to a gas-solid coexistence, which has previously been observed in hard-sphere systems with added dipolar interactions. ${ }^{27}$ This re-entrant, field-induced disorder-order-disorder transition is particularly visible in the Fourier transforms of the images, shown in the insets of Fig. 3.

As is normal in microscopy, the resolution along $Z$ is worse than the $X Y$ resolution. When coupled with the substantial refractiveindex mismatch between particles and medium and the overlap in fluorescence within a string of touching and fully fluorescent particles, this makes it challenging to obtain a fully reconstructed $3 \mathrm{~d}$ image and establish the nature of the crystalline state observed at an intermediate field strength (Fig. 3, middle row). We therefore performed experiments with the field along the $Z$ direction, perpendicular to the image plane. In this case the samples were contained between two ITO-coated cover glasses separated by a $70 \mu \mathrm{m}$ spacer. Fig. 4 (image plane in $X-Y$ and the field $E=0.23 \mathrm{~V}_{\text {rms }}$ $\mu \mathrm{m}^{-1}$ along the $Z$ direction) shows that the order is polycrystalline at best. However, it unambiguously demonstrates the presence of tiny crystallites with a square unit cell (shown in Fig. 4C).

The unit cell spacing in the $X-Y$ plane with the field along $Z$, extracted from Fig. 4, is $a=b=\sqrt{ } 2 \times 0.95=1.34 \mu \mathrm{m}$. The average nearest neighbor distance extracted from Fig. 3 (with the field along $X Y$ ) is $c=1.14 \mu \mathrm{m}$. A test for 4-fold bond orientational order yields approximately $40 \%$ of the particles showing square symmetry in the plane perpendicular to the field direction (calculated using a method reported elsewhere ${ }^{39}$ ). The inability to form crystallites over many inter-particle distances could be related to a lowering of the stability of the BCT crystalline state, due to both chain undulations along the field direction and the intrinsic polydispersity of compressible particles. The latter has been addressed in a theoretical study of polydisperse dipolar spheres. ${ }^{40,41}$ 


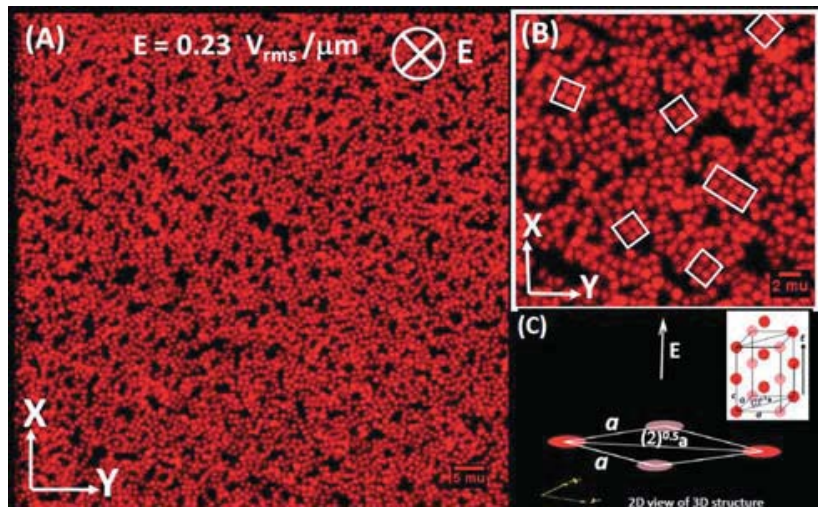

Fig. 4 (A) Image of a sample at $\phi_{\text {eff }}=0.85$ and $E=0.23 \mathrm{~V}_{\text {rms }} \mu \mathrm{m}^{-1}$ applied along the $Z$-direction using a $3 \times$ zoom. Objective: $63 \times$ oil immersion. (B) Expanded sub-region highlighting small crystallites (squares in the $X-Y$ plane) coexisting with voids. (C) Schematic representation of the BCT unit cell and its square base in the $X-Y$ image plane.

Next, we probed field-induced structural ordering at ultra-high volume fractions $\phi_{\text {eff }}=2.0$, where $a_{\mathrm{s}} / \sigma=0.68<1$ in the absence of an applied field. Interestingly, we found field induced aggregates without chain formation, and with no clear anisotropy, already at $E=0.06$ $\mathrm{V}_{\mathrm{rms}} \mu \mathrm{m}^{-1}$ (Fig. 3, bottom row). Specifically, there is a transition from a zero-field amorphous state (with dynamics quantified in the ESI, Fig. S2 $\dagger$ ) to a heterogeneous disordered state at very small fields which does not evolve significantly over experimental times. The origin of what is likely to be a reversible field-induced arrested phase separation is currently unknown, but is likely related to the fact that the zero-field nearest-neighbour distance is $0.68 \sigma$, i.e. much smaller than the core diameter of the microgels (Fig. 2A, inset), resulting in both much steeper repulsions and more complex field-induced interactions. Similar behavior was also seen at $\phi_{\text {eff }}=1.6$ (this is shown in the ESI, Fig. S4 $\uparrow$ ).

In summary, we have experimentally characterized the phase behavior of microgel suspensions under the application of an alternating electric field at different volume fractions (Fig. 1) for the first time. At low packing the field-induced structures are similar to those seen in hard-sphere colloids, but even here the low threshold value for string formation is a surprise. At high packing, we see at $\phi=0.85$, a novel field-induced transition from an amorphous state to an ordered solid state, and at $\phi=2.0$, a transition to a field-induced disordered state that shows no signs of anisotropy.

This work was supported by the Swiss National Science Foundation, the Swedish Research Council (Project 621-2011-4338) and the Natural Sciences and Engineering Research Council of Canada (NSERC). We gratefully acknowledge Christos Likos for illuminating discussions, Suresh K. Bhat for temperature-dependent DLS measurements of the particle size, and Andrew Morrow for help with the analysis of an image.

\section{References}

1 D. M. Heyes and A. C. Branka, Soft Matter, 2009, 5, 2681.

2 Z. Zhang, N. Xu, D. T. N. Chen, P. Yunker, A. M. Alsayed, K. B. Aptowicz, P. Habdas, A. J. Liu, S. R. Nagel and A. G. Yodh, Nature, 2009, 459, 230.
3 D. Gottwald, C. N. Likos, G. Kahl and H. Löwen, Phys. Rev. Lett., 2004, 92, 068301 .

4 D. Gottwald, C. N. Likos, G. Kahl and H. Löwen, J. Chem. Phys., 2005, 122, 074903.

5 C. N. Likos, H. Löwen, M. Watzlawek, B. Abbas, O. Jucknischke, J. Allgaier and D. Richter, Phys. Rev. Lett., 1998, 80, 4450.

6 E. Zaccarelli, C. Mayer, A. Asteriadi, C. N. Likos, F. Sciortino, J. Roovers, H. Iatrou, N. Hadjichristidis, P. Tartaglia, H. Löwen and D. Vlassopoulos, Phys. Rev. Lett., 2005, 95, 268301.

7 H. Senff and W. Richtering, J. Chem. Phys., 1999, 111, 1705.

8 M. Stieger, W. Richtering, J. S. Pedersen and P. Lindner, J. Chem. Phys., 2004, 120, 6197.

9 M. Das, H. Zhang and E. Kumacheva, Annu. Rev. Mater. Res., 2006, 36, 117.

10 D. A. Sessoms, I. Bischofberger, L. Cipelletti and V. Trappe, Philos. Trans. R. Soc., A, 2009, 367, 5013-5032.

11 Z. Meng, J. K. Cho, V. Breedveld and L. A. Lyon, J. Phys. Chem. B, 2009, 113, 4590 .

12 F. Scheffold, P. Diaz-Leyva, M. Reufer, N. B. Braham, I. Lynch and J. L. Harden, Phys. Rev. Lett., 2010, 104, 128304.

13 A. Fernandez-Nieves, H. Wyss, J. Mattsson and D. A. Weitz, Microgel Suspensions: Fundamentals and Applications, Wiley \& Sons, 1st edn, 2011.

14 P. S. Mohanty and W. Richtering, J. Phys. Chem. B, 2008, 112, 14692.

15 U. Gasser, B. Sierra-Martin and A. Fernandez-Nieves, Phys. Rev. E: Stat., Nonlinear, Soft Matter Phys., 2009, 79, 051403.

16 C. Casagrande, P. Fabre, E. Raphael and M. Veyssie, Europhys. Lett., $1989,9,251$

17 S. Granick, S. Jiang and Q. Chen, Phys. Today, 2009, 62, 68.

18 E. Bianchi, R. Blaak and C. N. Likos, Phys. Chem. Chem. Phys., 2011, 13, 6397.

19 S. Fraden, A. J. Hurd and R. Meyer, Phys. Rev. Lett., 1989, 63, 2320.

20 R. Tao and J. Sun, Phys. Rev. Lett., 1990, 65, 2820.

21 J. Martin, J. Odinek, T. Halsey and R. Kamien, Phys. Rev. E: Stat. Phys., Plasmas, Fluids, Relat. Interdiscip. Top., 1998, 57, 756.

22 U. Dassanayake, S. Fraden and A. van Blaaderen, J. Chem. Phys., 2000, 112, 3851 .

23 A. Yethiraj and A. van Blaaderen, Nature, 2003, 421, 513.

24 M. E. Leunissen, H. R. Vutukuri and A. van Blaaderen, Adv. Mater., 2009, 21, 3116-3120.

25 T. Halsey and W. Toor, Phys. Rev. Lett., 1990, 65, 2820.

26 A. Hynninen and M. Dijkstra, Phys. Rev. E: Stat., Nonlinear, Soft Matter Phys., 2005, 72, 051402.

27 A. Yethiraj, A. Wouterse, B. Groh and A. van Blaaderen, Phys. Rev. Lett., 2004, 92, 058301.

28 T. Hamanaka and A. Onuki, Phys. Rev. E: Stat., Nonlinear, Soft Matter Phys., 2007, 75, 041503.

29 E. Sanz, C. Valeriani, E. Zaccarelli, W. C. K. Poon, P. N. Pusey and M. E. Cates, Phys. Rev. Lett., 2011, 106, 215701.

30 C. P. Royall, E. C. M. Vermolen, A. van Blaaderen and H. Tanaka, J. Phys.: Condens. Matter, 2008, 20, 404225.

31 P. Yunker, Z. Zhang, K. B. Aptowicz and A. G. Yodh, Phys. Rev. Lett., 2009, 103, 115701.

32 M. Parthasarathy and D. Klingenberg, Mater. Sci. Eng., 1996, R17, 57.

33 W. Wen, X. Huang, S. Yang, K. Lu and P. Sheng, Nat. Mater., 2003, 2, 727 .

34 S. Chen, X. Huang, N. F. A. van der Vegt, W. Wen and P. Sheng, Phys. Rev. Lett., 2010, 105, 046001.

35 H. Ko, Z. Zhang, Y.-L. Chueh, E. Saiz and A. Javey, Angew. Chem., Int. Ed., 2010, 49, 616.

36 G. Romeo, L. Imperiali, J.-W. Kim, A. Fernández-Nieves and D. A. Weitz, J. Chem. Phys., 2012, 136, 124905.

37 A. van Blaaderen and P. Wiltzius, Science, 1995, 270, 1177.

38 A. D. Dinsmore, E. R. Weeks, V. Prasad, A. C. Levitt and D. A. Weitz, Appl. Opt., 2001, 40, 4152.

39 M. J. McDonald, A. Yethiraj and L. Y. Beaulieu, Meas. Sci. Technol., 2012, 23, 045606.

40 B. J. C. Cabral, J. Chem. Phys., 2000, 112, 4351.

41 H. Sun and K. W. Yu, Phys. Rev. E: Stat., Nonlinear, Soft Matter Phys., 2003, 67, 011506 